\title{
Big Data und Künstliche Intelligenz in Hydrologie und Wasserwirtschaft
}

Big Data und Künstliche Intelligenz sind aktuell weit verbreitete Schlagworte. Dies betrifft nicht nur verschiedenste Wissenschaftsbereiche, sondern auch aktuelle politische Diskussionen, wenn es z. B. um autonomes Fahren oder die umfassende Digitalisierung in der Industrie (Industrie 4.0) geht. Wir möchten zunächst die beiden Begrifflichkeiten Big Data und Künstliche Intelligenz (KI) im Kontext der Umwelt- und Geowissenschaften etwas ausführlicher beleuchten, bevor wir dann einen kurzen Überblick darüber geben, wie diese bereits aktuell Eingang in die Hydrologie und Wasserwirtschaft gefunden haben.

Big Data wird oft als Sammelbegriff für die enorm großen Datenmengen verwendet, die über moderne digitale Technologie erfasst und verfügbar gemacht werden können. Neben den automatischen hydrometeorologischen Messstationen mit elektronischer Datenübertragung sind es vor allem die Entwicklungen im Bereich der satellitengestützten Erdbeobachtung, die seit den 70er-Jahren des letzten Jahrhunderts große Fortschritte gemacht haben. Allein die Satelliten des aktuellen europäischen Copernicus-Programms erzeugen mit ihren hochauflösenden Instrumenten ein tägliches Datenvolumen von ca. 20 Terabyte, was einem HD Film von ca. 1,5 Jahren Länge entspricht. Diese großen Datenmengen können längst nicht mehr per Hand oder mit einfachen statistischen Verfahren ausgewertet und analysiert werden. Moderne Methoden aus dem Bereich der KI und des Maschinellen Lernens $(M L)$ kommen dabei vermehrt zum Einsatz und deren Weiterentwicklung ist in weiteren Bereichen der Wissenschaft und Industrie aktueller Forschungsschwerpunkt.

Für den Begriff Intelligenz an sich bestehen verschiedene (vage) Definitionen. So kann Intelligenz als die Fähigkeit beschrieben werden, in komplexen Situationen oder Umgebungen zurechtzukommen, d. h. die Situation ganzheitlich zu erfassen und fallweise zu lösen und zu bewältigen. $K I$ bezeichnet den Versuch, menschliche Entscheidungsstrukturen automatisiert ab- bzw. nachzubilden. Beispiele dafür sind die oben erwähnten autonomen Fahrzeuge, Industrieroboter, automatisierter Börsenhandel oder Sprach- und Bilderkennung. $M L$-Verfahren gelten als Teilbereich der $K I$ und bezeichnen Methoden zur computergestützten Generierung von Wissen aus beobachteten bzw. gemessenen Daten und persönlicher Erfahrung. Die entsprechenden Algorithmen lernen aus Beispieldaten und können diese nach Beendigung der Lernphase verallgemeinern, indem sie Muster und Gesetzmäßigkeiten in den Daten identifizieren. Dieser Vorgang ist im Prinzip mathematisch sehr ähnlich zum Kalibrieren eines hydrologischen Modells oder der Schätzung von Parametern eines linearen Regressionsmodells. Die zur Anwendung kommenden Methoden sind sehr vielfältig und einige der wichtigsten Verfahren werden in den folgenden Beiträgen näher vorgestellt. Einen wichtigen Teilbereich der ML-Verfahren stellen die sogenannten Deep-Learning ( $D L$ )-Verfahren dar. Sie basieren auf Neuronalen Netzen und der Begriff „Deep“ bezieht sich nicht auf ein „tieferes“ Lernen bzw. Verständnis, sondern beschreibt die Komplexität und Tiefschichtigkeit der verwendeten Netze. DL-Methoden haben, angetrieben von Technologieunternehmen wie Google oder Microsoft, den Bereich der Sprach- und Bilderkennung in den letzten 10 Jahren revolutioniert und können in angepasster Form auch sehr erfolgreich zur Beschreibung der Niederschlags-Abfluss-Dynamik, von Regionalisierungsstrategien oder der räumlich verteilten Verdunstung eingesetzt werden.

Im Folgenden werden zunächst in einem Beitrag von Klingler et al., als ein Beispiel für Big Data, die für den Oberen Donauraum verfügbaren Datensätze zu Niederschlag und Abfluss von den hydrometeorologischen Diensten, physio-geografische Einzugsgebietscharakteristika sowie Satelliten- und 


\section{Editorial}

Reanalyseprodukten zusammengestellt, analysiert und verfügbar gemacht. Diese Art der Datenbereitstellung und -standardisierung ist Teil aktueller globaler Initiativen im Bereich der hydrologischen Forschung. Dieser Datensatz dient u.a. als Basis für eine Anwendung von ML- und DL-Verfahren, deren Anwendung grundsätzlich von zur Verfügung stehenden Grundlagendaten abhängt.

Kratzert et al. zeigen im zweiten Beitrag das Potenzial sogenannter Long-Short-Term-Memory (LSTM)Netze, um einerseits die Niederschlags-Abfluss-Dynamik aus den gegebenen Daten zu „lernen“, aber auch erfolgreich Anwendungen in „unbeobachteten“ Gebieten zu übertragen. In ihrem Beitrag weisen die Autoren ebenfalls darauf hin, dass Neuronale Netze nicht als völlige „Black Box“-Systeme betrachtet werden müssen, sondern dass netzinterne Speicherzustände durchaus mit gemessenen Zustandsgrößen in der Natur korrelieren.

Feigl et al. nutzen in ihrem ersten Beitrag DL-Techniken im Rahmen der Regionalisierung von Modellparametern eines räumlich verteilten Modellsystems zur Beschreibung der N-A-Dynamik und Wasserhaushaltskomponenten. Die Regionalisierungsstrategie basiert auf dem Konzept der Transferfunktion, allerdings werden sowohl die mathematische Struktur der Transferfunktionen als auch deren Parameter aus beobachteten Abflussdaten und Zustandsgrößen gleichzeitig geschätzt. Das Verfahren nutzt Konzepte aus dem Bereich der Symbolischen Regression und integriert einen Variational Autoencoder als DL-Komponente zur Beschreibung der mathematischen Strukturen der Transferfunktionen.

Brenner et al. zeigen im vierten Beitrag, wie die LSTM-Netzwerkstruktur für die räumlich verteilte Abschätzung von Verdunstungsraten unter Ausnutzung globaler Datensätze erfolgreich genutzt werden kann.

Im fünften Beitrag vergleichen Feigl et al. verschiedene ML- und DL-Verfahren in Bezug auf ihre Fähigkeit die Temperatur von Fließgewässern für 10 Einzugsgebiete aus einer Anzahl von verfügbaren hydrometeorologischen Daten zu beschreiben.

Abschließend demonstrieren Sappl et al., wie erfolgreich ML-Verfahren und insbesondere der genutzte Temporal Fusion Transformer im Bereich der Siedlungswasserwirtschaft zur Vorhersage der Biogasproduktion in Faultürmen eingesetzt werden können.

Alle hier vorgestellten Arbeiten zeigen das enorme Potenzial, das die verschiedenen Verfahren der $\mathrm{KI}$ im Bereich der Hydrologie und Wasserwirtschaft (inkl. der Siedlungswasserwirtschaft) haben können. Man sollte sich aber grundsätzlich nicht von dem Begriff „Künstliche Intelligenz“ täuschen bzw. verführen lassen und glauben, dass einzig über deren Verwendung Problemlösungen ohne Einschränkungen möglich sind. Die Verfahren benötigen in der Regel große Datenmengen, um darin enthaltene Prozesse, Muster und Strukturen identifizieren und generalisieren zu können. Sind die entsprechenden Daten vorhanden, dann können diese Verfahren sehr effizient und erfolgreich eingesetzt werden.

Auch wenn es unumstritten ist, dass sich die vorgestellten Methoden ausgezeichnet für die Zeitreihenvorhersage eigenen, ist eine lebhafte wissenschaftliche Diskussion im Gange, inwiefern diese Verfahren unser (Prozess-)Verständnis und Wissen an sich vertiefen können und wie der Nutzen für die Wissenschaft zu beurteilen ist. Eine gute Zeitreihenvorhersage mit KI bedeutet nicht unbedingt, dass die dahinterliegenden Prozesse verstanden werden. Die Kombination beider Welten - KI und klassische prozessbasierte Modelle - können hier die Grundlage für weitere Forschungen sein. 
Univ.-Prof. Dipl.-Geo-

ökol. Dr. K. Schulz (ه) •

DI Dr. M. Herrnegger ( $ه)$

Institut für Hydrologie und Wasser-

wirtschaft, Universität für Bodenkultur

Wien,

Muthgasse 18,

1190 Wien, Österreich

karsten.schulz@boku.ac.at;

mathew.herrnegger@boku.ac.at 